\title{
Bioanalytics and higher order electrokinetics
}

\section{Mark A. Hayes $^{1}$ (D) Federica Caselli ${ }^{2}$}

Published online: 14 May 2020

(C) Springer-Verlag GmbH Germany, part of Springer Nature 2020

We are at the cusp of a revolution of discovery and creation for understanding and applying electric fields to manipulate and characterize materials on the micro- and nanoscale. Enabled by a range of fabrication techniques and ingenious use of existing materials, linear and simple forces are coupled with higher order and complex interactions that are only now being discovered and explored. The types of materials which can be addressed and studied are large: any particle placed in solution will develop electrophysical properties which can be used for selective differentiation. While inorganic and simple organic materials are of great interest, especially during the early development of dielectrophoresis, for instance, by far the most energy is being focused on biological cells and particles. In fact, all molecules and particles (bioparticles: cells, organelles, exosomes, protein complexes, viruses, and proteins) have appreciable 'higher order' electric field interactions. These higher order properties include permanent dipole, quadrupole and higher order moments, along with overall and interfacial polarizabilities. Typically, only the monopole moment (Coulombic) of atoms, molecules and particles in solution have been exploited (electrophoresis in all of its forms). We are at an inflection point where the higher order and complex interactions will be both a subject of intense study and their development such that they become a new and reliable tool in the study and manipulation of matter.

Published in the topical collection Bioanalytics and Higher Order Electrokinetics with guest editors Mark A. Hayes and Federica Caselli.

Mark A. Hayes

mhayes@asu.edu

Federica Caselli

caselli@ing.uniroma2.it

1 School of Molecular Sciences, Arizona State University, Mail Stop 1604, Tempe, AZ 85287, USA

2 Department of Civil Engineering and Computer Science, University of Rome Tor Vergata, Via del Politecnico 1, 00133 Rome, Italy
The fields which are contributing to this area are varied. They include nanoscience/nanotechnology, analytical chemistry, applied physics, biochemistry, instrumentation development, materials science, electrical engineering, biophysics and physical chemistry. These topics are the result of a Web of Science search for 'dielectrophoresis' and the list should be much bigger, including neuroscience, microbiology, medical science, environmental, space exploration, oceanography, modeling and simulations, signal and image processing, artificial intelligence/machine learning, and bioengineering.

As Co-Guest Editors, we were honored to have such an enthusiastic response to our request for manuscripts and the large variety of fields of study reflect the growing depth and breadth of non-linear electric field research. This issue provides a stochastic sampling, if you will, of the breadth of work being done across the world. From immobilizing small molecules with combined non-linear forces to characterizing several species of bacteria by their dielectrophoretic properties are presented here. In constructing this issue, we wanted to allow for a truly broad sampling and this resulting issue certainly reflects this element.

This issue has works aimed at traditionally poorly understood and difficult targets. Exploiting a variety of non-linear, electric field/gradient-induced processes, Laux et al. demonstrate effective capture of small molecules spread across the electrode surface, as opposed to being captured at the edges only. The performance of dielectrophoresis has always been predicted to be quite weak on particles the size of proteins, but recent theoretical work has caused a re-examination of this premise. Hayes examines the previous experimental evidence and compares these results to traditional and emerging theoretical constructs.

A fundamental and foundation effect is possible changes at the electrode surface caused by application of AC voltage in electrode-based dielectrophoresis and an elegant study is presented by Heineck et al. showing significant changes to platinum electrodes under common conditions for dielectrophoretic studies. In distinct contrast, contactless dielectrophoresis is an attractive approach to reduce the risk 
of electrode-induced cell damage, however it may suffer from microfabrication issues. To overcome this limitation, Salahi et al. report an original strategy to microfabricate selfaligned multilayered microfluidic devices enabling reproducible contactless dielectrophoresis.

Hill and Lapizco-Encinas have adapted parallel post-based insulator dielectrophoresis to chromatographic capabilities, showing data from 1 to $10 \mu \mathrm{m}$ diameter particles and even same-sized particles with differing surface charge. Significant advances in understanding and performance of particle filtration at the industrial scale using dielectrophoretic forces are shown by Lorenz et al. Using microporous ceramic materials they show compelling evidence that well-matches the theoretical construct underlying the filtration process.

Besides electrorotation and dielectrophoresis, the interaction of AC electric fields with biological matter is also the basis of impedimetric biosensing, a label-free characterization technique suited to both microfluidic systems and cell culture systems. Honrado et al. present a novel impedance-based microfluidic system for the multiparametric characterization of single cells flowing at high throughput. As a peculiar feature of this work, machine learning is exploited to analyze the recorded electrical fingerprints, showing accurate characterization of thousands of red blood cells and yeasts with a unitary prediction time of $0.4 \mathrm{~ms}$. Liu et al. used impedimetric sensing for label-free dynamic monitoring of interactions between cells of placental origin and malaria-infected erythrocytes in a placental malaria model. They were able to show a correlation between impedance changes and cellular and molecular evidence of cell proliferation, syncytial fusion and inflammatory response.

Epping et al. used whole cell catalysis as the driver for investigating the biophysical effects of isopropyl alcohol on E. coli using AC dielectrophoresis and insulating posts. They were able to show distinct and quantifiable changes with a novel dielectrophoresis method after short exposure to low concentrations of the alcohol. An important challenge of DEP-based cell characterization systems is throughput.

Torres Castro et al. present a microfluidic device to characterize the DEP behavior of single flowing cells at high throughput and without the need for sample dilution, by using a combination of planar electrodes, localized constrictions and flow focusing.

With rising concerns over antibacterial resistance, the development of rapid and portable detection systems becomes of increasing interest. Coll De Peña et al. propose the creation of an electrokinetic library for the identification of microorganisms such as bacterial and yeast cells, based on the novel concept of the electrokinetic equilibrium condition. Among various dielectrophoretic approaches, isomotive dielectrophoresis has the unique ability to provide uniform dielectrophoretic (iso-DEP) force. Rashed and Williams discuss the current status and present challenges of iso-DEP, with particular emphasis on platform design and applications, and provide their view on future directions.

In receiving, reading and reviewing these manuscripts, we were struck by the novel groundbreaking work being presented. These papers move each field forward and in some cases by a substantial amount. Our special thanks go out to the reviewers (who were very timely in their response!) and to the Editorial Office and Editors for this opportunity and support throughout the process. We are very proud and, again, honored, to have been able to help bring this Topical Collection issue to you.

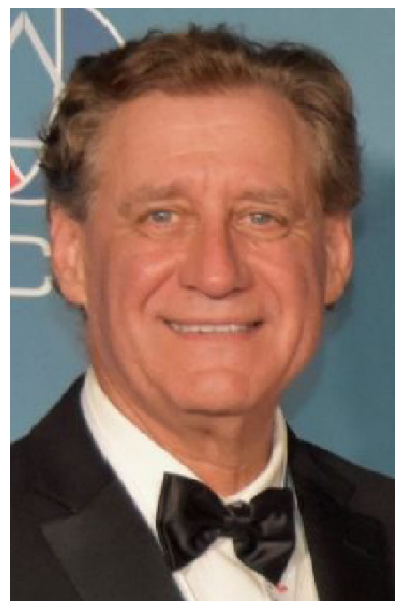

Mark A. Hayes is Professor in the School of Molecular Sciences at Arizona State University in Tempe, Arizona (USA) and W.W. Clyde Visiting Chair, College of Engineering, University of Utah in Salt Lake City, Utah (USA). His current work is focused on ultrahigh resolution separation and concentration of biomolecules, bioparticles and cells using microfluidic approaches, commonly employing electric fields.

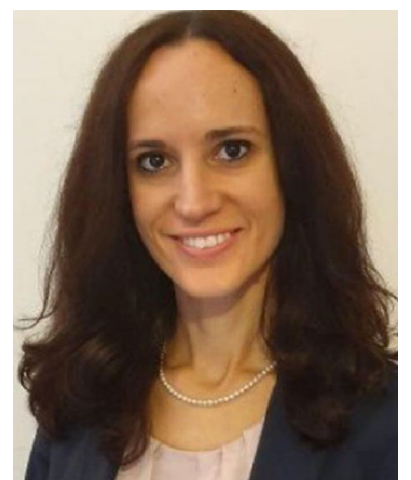

Federica Caselli is Associate Professor in Biomedical Engineering at the University of Rome Tor Vergata. She graduated with a degree in medical engineering and mathematics, and obtained her Ph.D. in advanced computational methods in biomechanics. Her research deals with the development of lab-on-a-chip devices for diagnostics and life science, with a special focus on microfluidic impedance cytometry for single-cell biophysical phenotyping. She has published about a hundred contributions in journals, conference proceedings, and book chapters, and she was awarded a grant from the "Scientific Independence of Young Researchers Programme." 